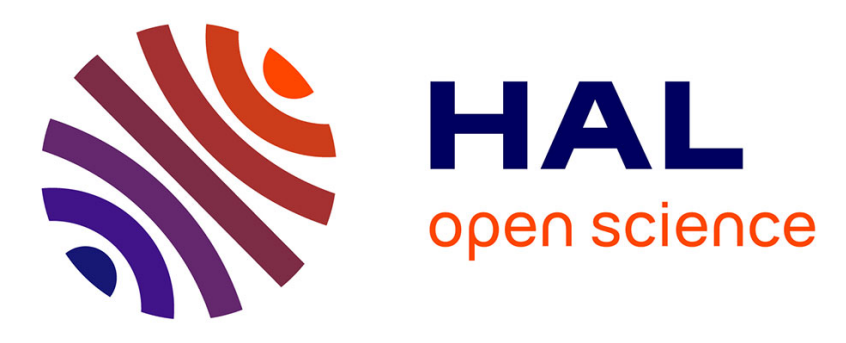

\title{
Groups in Space: Stereotypes and the Spatial Agency Bias
}

\author{
Anne Maass, Caterina Suitner, Xenia Favaretto, Marina Cignacchi
}

\section{To cite this version:}

Anne Maass, Caterina Suitner, Xenia Favaretto, Marina Cignacchi. Groups in Space: Stereotypes and the Spatial Agency Bias. Journal of Experimental Social Psychology, 2009, 45 (3), pp.496. 10.1016/j.jesp.2009.01.004 . hal-00664189

\section{HAL Id: hal-00664189 \\ https://hal.science/hal-00664189}

Submitted on 30 Jan 2012

HAL is a multi-disciplinary open access archive for the deposit and dissemination of scientific research documents, whether they are published or not. The documents may come from teaching and research institutions in France or abroad, or from public or private research centers.
L'archive ouverte pluridisciplinaire HAL, est destinée au dépôt et à la diffusion de documents scientifiques de niveau recherche, publiés ou non, émanant des établissements d'enseignement et de recherche français ou étrangers, des laboratoires publics ou privés. 


\section{Accepted Manuscript}

Groups in Space: Stereotypes and the Spatial Agency Bias

Anne Maass, Caterina Suitner, Xenia Favaretto, Marina Cignacchi

PII:

S0022-1031(09)00006-7

DOI:

10.1016/j.jesp.2009.01.004

Reference:

YJESP 2200

To appear in:

Journal of Experimental Social Psychology

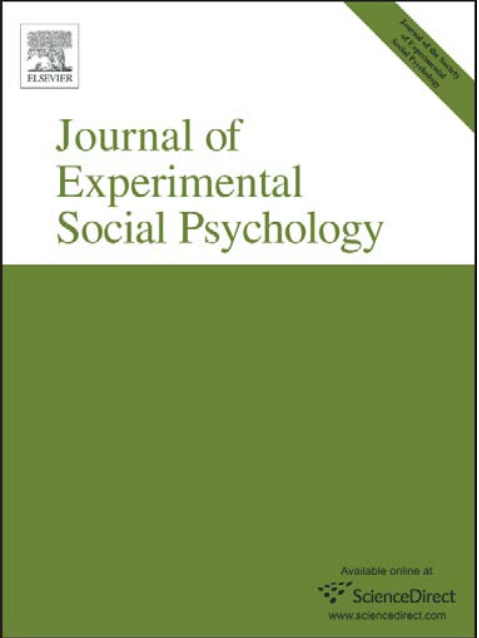

Received Date:

13 September 2007

Revised Date:

19 September 2008

Please cite this article as: Maass, A., Suitner, C., Favaretto, X., Cignacchi, M., Groups in Space: Stereotypes and the Spatial Agency Bias, Journal of Experimental Social Psychology (2009), doi: 10.1016/j.jesp.2009.01.004

This is a PDF file of an unedited manuscript that has been accepted for publication. As a service to our customers we are providing this early version of the manuscript. The manuscript will undergo copyediting, typesetting, and review of the resulting proof before it is published in its final form. Please note that during the production process errors may be discovered which could affect the content, and all legal disclaimers that apply to the journal pertain. 


\title{
Groups in Space:
}

Stereotypes and the Spatial Agency Bias

Anne Maass

\author{
Caterina Suitner Xenia Favaretto
}

Marina Cignacchi

University of Padova

Please, send correspondence regarding this ms. to:

Anne Maass

DPSS, University of Padova

Via Venezia 8

35139 Padova, Italy

anne.maass@unipd.it 
Abstract

We propose that spatial imagery is systematically linked to stereotypic beliefs, such that more agentic groups are envisaged to the left of less agentic groups. This Spatial Agency Bias was tested in three studies. In Study 1, a content analysis of over 200 images of malefemale pairs (including artwork, photographs, and cartoons) showed that males were overproportionally presented to the left of females, but only for couples in which the male was perceived as more agentic. Study $2(\mathrm{~N}=40)$ showed that people tend to draw males to the left of females, but only if they hold stereotypic beliefs that associate males with greater agency. Study $3(\mathrm{~N}=61)$ investigated whether scanning habits due to writing direction are responsible for the Spatial Agency Bias. We found a tendency for Italian-speakers to position agentic groups (men and young people) to the left of less agentic groups (females and old people), but a reversal in Arabic speakers who tended to position the more agentic groups to the right. Together, our results suggest a subtle spatial bias in the representation of social groups that seems to be linked to culturally determined writing/reading habits. 
Events, including social events, evolve in time and space. The same holds for mental images that generally have a temporal and a spatial component. Regardless of whether we imagine armies going to war or children playing, we envisage their actions embedded in space. Whereas space is an essential topic in other fields of psychology, including cognitive psychology, neuro-psychology, and psychology of the arts, social psychologists have generally dedicated little attention to spatial aspects of social cognition, with the possible exception of recent theorizing on embodied cognition (e.g., Barsalou, 1999; Richardson, Spivey, Barsalou and McRae, 2003; for a review see Smith, L.B., 2005) and on the face-ism effect that focuses on the social implications of showing proportionally more face than body in a person's visual representation (e.g., Archer, Iritani, Kimes, \& Barrios, 1983; Schwartz and Kurz, 1989; Kolbe \& Albanese, 1996).

Although generally ignored by social psychologists, spatial biases may have interesting consequences for social cognition. In fact, spatial arrangements may represent a subtle way to communicate differences between groups, thereby contributing to the maintenance of the status quo of the existing social structure.

In this article, we will focus on the horizontal dimension and ask which groups will occupy the left vs. right position in our mental images. We will argue that people think of individuals and groups as located in space and that the imagined spatial relations between them are by no means arbitrary, but reflect stereotypic beliefs related to agency. We will first briefly review relevant findings on horizontal spatial asymmetries reported in the cognitive literature, followed by a discussion of theoretical explanations. We will then introduce the Spatial Agency Bias (SAB) according to which stereotypically agentic groups are preferentially located to the left of less agentic groups (see Chatterjee, 2002) and report three studies testing this hypothesis. 
Groups in space

\section{Spatial biases in the processing of physical stimuli}

A very robust and pervasive finding, emerging from different research paradigms in cognitive and neuro-psychology, is the tendency to imagine events preferentially as evolving from left to right, at least in Western cultures. For example, when asking people to draw scenes corresponding to minimal subject-verb-object phrases (e.g., Fabio feeds the dog), a vast majority of respondents will position the sentence subject (Fabio) to the left and the sentence object (dog) to the right (Chatterjee, Southwood, \& Basilico, 1999). In a similar vein, inhibition of return, that is the tendency to orient attention to novel locations rather than to return to previously attended locations, is stronger when the sequence of stimuli have a left-to-right (LR) trajectory (Spalek \& Hammad, 2004). Similar asymmetries emerge for the representational momentum phenomenon, namely the tendency to memorize the final position of a moving object as slightly further along the implied path (Freyd \& Finke, 1984; Hubbart, 2005). Again, this systematic error is stronger for LR motion (Halpern \& Kelly, 1993). Also, research on the imaginary number line (with small numbers envisaged to the left, large numbers to the right, see SNARC effect, Dehaene, Bossini \& Giraux, 1993) suggests a LR trajectory. The same is true for studies on the perception of time showing that, in LR writing cultures, time is envisaged as flowing from LR (Gevers, Reynvoet, \& Fias, 2004; Tversky, Kugelmass, \& Winter, 1991; Borodisky, 2001; Santiago, Pérez, Lupiáñez, \& Funes, 2007). Interestingly, research on art appreciation shows a very similar pattern, with observers focussing initially on the left side of their visual field and subsequently shifting attention from

L to R (Heron, 1957; Elkind \& Weiss, 1967).

Together, these and other findings suggest that observers explore space with a LR trajectory and process physical stimuli easier when they follow a LR (rather than RL) vector, at least in cultures in which language is read/written from LR. Why should this be the case? Theoretical explanations of spatial asymmetries 
Groups in space

In many of these different areas of research, hemispheric specialization was initially considered a strong candidate for explaining the obtained results (e.g., Chatterjee et al., 1999, 2001; Jackendoff, 1996). However, cross-cultural research has often found total, or at least partial, reversals in cultures where languages are written from right to left (RL), such as Arabic, Hebrew, or Urdu, suggesting that language-related scanning habits play an important role in such spatial asymmetries. Indeed, reversals have been reported in many different areas of psychology and on different tasks, including inhibition of return (Spalek \& Hammad, 2004), representational momentum (McBeath, Morikava, \& Kaiser, 1992), imaginary number line (Dehaene, Bossini, \& Giraux, 1993), visual imaging of subject-verb-object sentences (Maass \& Russo, 2003), and drawing, exploration of art, and aesthetic preferences (e.g., Chokron \& De Agostini, 2000; Nachshon, 1985; Nachshon, Argaman, \& Luria, 1999; Tversky, Kugelmass, \& Winter, 1991). Some studies also suggest that culture-specific asymmetries in space perception and directional tendencies only occur after learning to read and to write, as in the case of Fagard and Dahamen's (2003) study comparing French and Tunisian children, suggesting that writing/reading habits are implicated in spatial bias.

Why should writing direction affect spatial processing in tasks that have little or nothing to do with writing? From an embodiment perspective, the very mechanism of writing and the visual scanning while reading lead to a generalized habit of exploring space in a specific direction (either LR or RL, depending on the language). Starting from elementary school age, people in developed countries spend a remarkable amount of their time reading and writing ${ }^{1}$. In this way, scanning habits become so pervasive that they create a general spatial scheme that then generalizes across tasks.

Besides the mechanical aspects of writing and the visual aspects while reading, there is an additional, linguistic factor that may contribute to a generalized spatial schema for action, namely the order in which agent and recipient are mentioned in standard active sentences (for a discussion of agency see Duranti, 2004). In Indo-European languages, the 
Groups in space

agent typically occurs in the subject (or nominative) position, at least in active sentences, whereas the patient occurs in the object (or accusative) position (e.g., Nausicaa teases her father). Obviously, there are numerous exceptions to this general rule (e.g., passive voice, intransitive verb phrases or strategical ordering of the words). However, as a general rule, in the majority of active phrases describing interpersonal actions, the agent is likely to occur in the subject position and the patient in the object position.

This has direct implications both for the time and the space dimension, considering that the sentence subject precedes the sentence object in most languages. With the exception of few languages spoken by relatively small language communities (Fijian, Malagasy, Xavante, Hixkaryana, Dyrbal), in the majority of known languages the subject precedes the object in standard active sentence, regardless of whether the verb is placed between subject or object (e.g, English, French, Chinese), whether it follows the object (e.g., Japanese, Turkish) or whether it precedes both subject and object (e.g., Hawaian). In terms of spoken language, this implies that the agent is mentioned before the patient, whereas spatially, this implies that in LR languages, the agent is likely to appear to the left of the patient, with the action flowing from LR. In line with this idea, simple (orally presented) subject-verb-object phrases are imagined and drawn in a way that the agent is positioned to the $\mathrm{L}$ of the patient (Chatterjee, Southwood \& Basilico, 1999). In languages like Arabic, Hebrew, Urdu or Farsi, the same temporal ordering of subject and object will lead to an opposite spatial arrangement, so that the action is likely to flow from $\mathrm{R}$ (agent) to L (patient). Indeed, in such languages the subject of subject-verb-object phrases is generally envisaged to the right of the object (Maass \& Russo, 2003). Thus, scanning habit and the standard ordering of subject (agent) and object (recipient/patient) may jointly contribute to the culture-specific spatial biases reviewed above. Extrapolating to the social domain: The Spatial Agency Hypothesis

Extrapolating from this general rule, we hypothesize that school children and adults raised in LR languages will form mental images that mirror the thematic role assignment of 
Groups in space

their language, thus envisaging more agentic individuals or groups to the left of less agentic ones. Chatterjee (2002), focusing on spatial orientation in portraits, was probably the first to propose a systematic link between stereotypes and spatial imaging. He hypothesized that, if there is a general LR scheme for action, then stereotypically more agentic targets should be portrayed facing R, less agentic targets facing L (as seen from the perspective of the observer). His account was able to explain why men are less likely to be portrayed facing left than are women who are overwhelmingly portrayed in that direction (Chatterjee, 2002; Humphrey \& Mc Manus, 1973; Gordon, 1974; Grüsser, Selke \& Zynca, 1988; ten Cate, 2002; Suitner \& Maass, 2007). According to Chatterjee this bias is the combined effect of stereotypes associating men with greater agency and a diffuse LR scheme for action.

Although Chatterjee's (2002) prediction was mainly concerned with head rotation in portraits, his argument can, in principle, be extended to any mental representation involving social groups that differ in agency. Decades of research on stereotype content demonstrates that most groups can be classified along two basic dimensions, namely competence, instrumentality, masculinity or agency on one side and warmth, expressiveness, femininity or communality on the other (Fiske, Cuddy, Glick, \& Xu, 2002; Fiske, Xu, Cuddy, \& Glick, 1999; Judd, James-Hawkins, Yzerbyt, Kashima, 2005). Emblematic is research on gender stereotyping (including self-stereotyping), showing that males are generally associated with higher levels of agency and activity than females (Abele, 2003; Bakan, 1966; Conway, Pizzamiglio \& Mount, 1996; Spence, Helmreich \& Stapp, 1974; Spence \& Helmreich, 1978, Else-Quest, Hyde, Goldsmith, and Van Hulle, 2006, for an overview). If males are perceived as more active than women, then it would not surprise if, in our mental images but also in representations such as art work, films etc, males occurred more often in the more agentic left position than females. Thus, our first prediction is that, following the general LR scheme of action in Western cultures, people will envisage intergroup situations so that the more agentic group (males) is envisaged to the left, the less agentic group (females) to the right. Second, 
Groups in space

this systematic bias, which we will refer to as Spatial Agency Bias (SAB), should be closely linked to stereotypic expectations, such that only people endorsing the agency-related stereotype should show the spatial bias.

\section{Overview of research}

In order to test this possibility, we conducted three studies. The first study consisted of two content analyses investigating images of male-female pairs (such as Adam and Eve) in order to test whether the male was over-proportionally portrayed to the left of the female. In the second study we investigated our hypotheses experimentally by asking lay people to draw scenes involving male and female teams. We also assessed their beliefs concerning gender differences, considering that SAB is predicted only for those who endorse traditional stereotypes. A final study intended to test the possible mechanisms driving the observed asymmetries, by comparing Italian-speakers whose native language is written/read from LR and Arabic-speakers whose native language is written/read from RL. If culturally determined scanning habits are responsible for the SAB, Italians and Arabs should show opposite biases.

\section{Study 1A: Adam and Eve}

The aim of our first study was to test the positioning bias regarding males and females in art production. The advantage of analyzing artwork is that, on one side, the hypothesis can be tested on already existing material that was created for reasons unrelated to psychological experimentation. On the other side, it offers a much wider historical perspective as it allows to go back in time and to investigate spatial positioning biases over centuries.

Different from previous research investigating head rotation in portraits of individual women or men, we were interested in the global spatial positioning in paintings in which both a male and a female were depicted. Assuming (a) that the more agentic person will occupy the left position and (b) that men are stereotypically perceived as more agentic than women, we expected males to have a higher likelihood than females to be placed on the left. To our knowledge, this question has rarely been investigated, an exception being the analysis of the 
Groups in space

Annunciation, showing the angel Gabriel together with the Virgin Mary. McManus (2005) reports that in $97 \%$ of the 209 paintings considered, the angel enters from the left (from the perspective of the observer) whereas the Virgin Mary is located to the right. Although consistent with the SAB hypothesis, this finding may either be due to the more active role of Gabriel in this specific scene, due to his sex, or both. To untangle these explanations, we decided to analyze paintings in which males and females display a similar degree of (in)activity (for example both standing).

In order to test this hypothesis, we selected art work of what we believe is the most emblematic representation of man and woman in Western civilization, namely Adam and Eve. We therefore analyzed images of the couple available on the web (Google Images), hypothesizing that Adam would be positioned to the left of Eve more often than vice versa. Since word-order regularities are rather common (McGuire \& McGuire, 1992) and are systematically linked to spatial representation (Chatterjee et al., 1999; Maass \& Russo, 2003), we varied the order in which Adam and Eve were mentioned in the search (Adam and Eve vs. Eve and Adam). If spatial positioning of Adam and Eve varied as a function of keyword order this would suggest that the bias is due to a linguistic order effect rather than to stereotype content concerning gender differences.

Method

Materials were selected through a Google Images search using "Adam and Eve" and "Eve and Adam" as key words (search done in January 2006, using the Italian version of Google). All 120 images appearing on the first 3 pages of the two searches were included in the analyses. After exclusion of images that could not be coded (such as abstract representations, animals, single individuals), the final material consisted of 90 representations of Adam and Eve of different nature, including paintings, frescos, photographs etc.. We coded the relative position in which Adam and Eve appeared in the composition, as seen from the observer's perspective. 
Groups in space

Results

In line with our hypothesis, we found that Adam appeared to the left of Eve in $62 \%$ $(f=56)$ of the images whereas Eve occupied the left position only in $38 \%(f=34)$ of the cases. A bi-variate log linear analysis showed that this gender effect is statistically significant, $\chi^{2}=$ 5.31, d.f. $=1, p=.02$. There was no interaction with word order, $\mathrm{Y}^{2}=1.34$, d.f. $=1, p=.25$. The positioning bias emerged in both keyword-ordering conditions although it was slightly weaker when Eve preceded Adam. Using the keywords "Adam and Eve", Adam occurred on the left in $67 \%(f=35)$ of the representations (vs. Eve's 33\%, $f=17)$, whereas using the keywords "Eve and Adam", Adam occurred on the left in 55\% $(f=21)$ of the images (vs. Eve's $45 \%, f=17$ ). Thus, there was no evidence that the spatial bias would reverse when using "Eve and Adam" as search criterion.

\section{Discussion}

The findings of this content analysis suggest that Adam, presumably the more agentic figure, appears predominantly to the left of the composition, confirming our main hypothesis. The effect due to word order in the Google research was partially excluded, but the possibility remains that the common order of naming the couple in European languages, with Adam mentioned before Eve, affected the positioning of Adam to the left of Eve.

\section{Study 1B: Addams, Flinstones, Simpsons}

We therefore tested a series of male-female pairs, that are not labeled with any specific word-order but that are generally referred to with an overarching label. For example, people generally refer to Groenings's famous cartoon characters of Springfield as "the Simpsons" rather than as "Marge and Homer" or as "Homer and Marge". We therefore analyzed still representations of three male-female couples in the media, namely the Simpsons the Flintstones, and the Addams. Again, the main hypothesis was that, overall, the male protagonist would be portrayed to the left of the female more frequently than would be expected by chance. 
Groups in space

We futher predicted that the SAB, will occur only when males are indeed associated with greater agency. We therefore tested the perceived agency of male and female in each couple predicting that the left positioning bias would only occur for those families in which the male is perceived - in line with traditional gender stereotypes - as more agentic than the female.

\section{Pilot resesarch}

We initially asked a pre-test sample $(\mathrm{N}=134)$, to rate 12 adjectives indicative of dominance (e.g., dominant), agency (e.g., active), and communion (e.g., affectionate) as typical of females vs. males on a $66 \mathrm{~mm}$ feeling thermometer while counterbalancing order of presentation and scale endpoints (male to left and female to right, or vice versa). A principal component analysis showed that the adjectives could be grouped into three main factors, accounting for $59 \%$ of the variance, that can be easily interpreted as Communality (cordial, altruistic, affectionate, compassionate, helpful, humble; alpha = .83), Agency (active, productive, efficient; alpha $=.67$ ) and Dominance (strong, dominant, authoritative, alpha = .69). Only the agency subscale is of theoretical relevance for the aims of this study.

We then asked a small sample of participants $(\mathrm{N}=13)$ who were "quite" or "very" familiar with each TV program, to rate all three families on the 12 items. Participants responded to each item (e.g., Who is more active?) on a 4-point scale in which the endpoints represented the male and female of each couple (e.g. Marge - Homer or Homer-Marge, counterbalanced).

An ANOVA on the mean agency ratings showed that the three families differed reliably in perceived agency, $F(2,24)=11.70, p<.001, \eta_{\mathrm{p}}^{2}=.49$. The male (relative to the female) was rated most agentic for the Addams family $(M=2.90)$ and least for the Simpsons $(M=1.51)$, with the Flintstones $(M=2.23)$ occupying an intermediate position. A pair-wise comparison (Bonferroni corrected), showed that the Addams are rated more agentic than the other two families, $p_{\mathrm{s}}<.05$, with no difference in agency ratings between Simpsons' and 
Groups in space

Flintstones' agency ratings, $p=.21$. Comparing the means for each family with the neutral scale-midpoint (no difference in agency between male and female of each family), only in the case of the Addams family was the male rated more agentic, one-sample $t(12)=2.88, p=$ .01 , whereas no difference emerged for the Flinstones, one-sample $t(12)=-.93$, n.s., and a reversal was found for the Simpsons, one-sample $t(12)=-5.00, p<.001$. This suggests that a left positioning of the male should only be expected for the Addams family.

Main study: Spatial positioning

For each family, we then examined the first 60 images in Google using "the Simpsons", "the Flintstones", and "the Addams" as key words. We simply recorded the number of times in which the male vs. the female of each couple occupied the left position (from the perspective of the observer).

Overall, males were positioned to the left in $60 \%$ of all images, which deviates reliably from chance, binomial test $p=.008$, thereby confirming our first hypothesis. However, looking separately at the families, this was entirely due to the Addams family where the male was presented to the left in $82 \%$ of all representations. Binomial tests comparing the distribution to the baseline probability of $50 \%$ indicated that only the Addams family deviated systematically from what would be expected by chance, $p<.001$. No systematic bias was found either for the Simpsons (males to left in 53\% of all cases) and the Flintstones (males to left in $43 \%$ of all cases). Thus, in line with the second hypothesis, the spatial agency bias was found only when the male was perceived as more agentic than the female.

\section{$\underline{\text { Discussion }}$}

Together, our first study suggests that people tend to position the more agentic person to the left when portraying two people of different activity levels. Thus, Adam was presented to the left of Eve and Gomez to the left of Mortissa in about 3 out of 4 images. In addition, Study $1 \mathrm{~b}$ also informs us about the role of stereotypic perceptions in the SAB, considering 
Groups in space

that the bias was strictly linked to how agentic the male and the female in each couple was perceived. The spatial agency bias was not found in the two cartoon couples in which male and female were perceived as equally agentic or in which perceptions were actually opposite to stereotypic expectancies, as in the case of Homer and Marge. One may wonder why the spatial pattern did not reverse for the Simpsons, given that Marge was rated as more agentic than Homer. We believe that the bias was possibly mitigated by two competing tendencies, At the social level, gender stereotypes prescribe that males are to be considered more agentic (thus placed to the left), but at the individual level it is Marge who is considered more agentic than Homer. The interplay between these two ways of conceptualizing agency may be responsible for the fact that spatial bias in the unconventional couple (Simpsons) was eliminated, but not reversed.

Obviously, content analyses of this sort have all kinds of limits. In particular, we cannot exclude that other, uncontrolled factors may have influenced the choices of professional cartoonists, artists, photographers, and the like. We therefore tested the spatial agency bias in a more controlled way in Study 2.

\section{Study 2: Women and men in competition}

In our second experiment, we investigated how lay people envisage interactions between males and females to evolve in space. Participants were given brief descriptions of hypothetical competitions between male and female teams, such as a chess tournament or a volleyball game. They were provided with a simple drawing of each scene and instructed to draw the players of the two teams in whatever position they imaged them to be. We also assessed gender stereotypes as we predicted spatial bias to mirror the participants' gender stereotypes so that only those who endorse the stereotype associating males with greater agency were expected to position men to the left, whereas this pattern was expected to disappear or even reverse for those who consider women more agentic.

\section{Method}


Groups in space

Participants. Twenty male and twenty females participants (mean age: 34 years), including 6 students and 34 employed, from Italy volunteered for this study. The majority (38) was right-handed; 17 participants had a high school, 15 a university degree.

Procedure and Material. The study was introduced as concerning how people imagine different events. Participants were asked to complete a 4-page booklet, each page portraying a scene in which a male and a female team were competing with each other. The four scenes were a card game, a ping-pong, a draughts, and a volleyball tournament. For each scene, participants received a simple drawing (for example a ping-pong table, a volleyball court, etc.) and were asked to add the two teams, indicating clearly where the two teams were positioned. Example: “In your neighborhood, a ping-pong tournament has been organized in which female teams compete with male teams. Please, draw the two teams indicating which team is which”. Each participant received two scenes in which males were mentioned first and two scenes in which females were mentioned first. The presentation order of the teams and of the scenes were fully counterbalanced. After completion, participants were debriefed about the purpose of the experiment and offered to receive a summary of the findings.

Agency vs. communion scale. Subsequently, the participants completed the Spence and Helmreich's (1978) Personal Attributes Questionnaire (PAQ), consisting of the agency (e.g.,: active, independent) and the communion sub-scales. Only the agency sub-scale is of interest to the present study. Whereas the original scale requires participants to rate themselves on each item, participants in the present study were asked to rate, on a 5 point scale $(1=$ not at all, $5=$ very much), the degree to which the item applied to men or women in general. Males rated first men and subsequently women on the 24 PAQ items, whereas females rated first women and then men. The agency sub-scale contained originally 16 items, one of which (competitive) was excluded because of its negative item-total correlation. The internal consistency of the scale was satisfactory (Cronbach's alpha $=.71$ when rating men, .75 when rating women $)^{2}$. 
Groups in space

\section{Results}

Perceived agency. We first tested whether males were, on the average, perceived as more agentic than females as would be suggested by research in gender stereotyping. A 2 (participant gender) x 2 (target gender) ANOVA with repeated measures on the second factor, using perceived agency as repeated measure, showed that, on the average, male $(M=3.15)$ and female targets $(M=3.14)$ were judged equally agentic, $F(1,38)=.01$, n.s. Also, ratings of male and female participants did not differ, $F(1,38)=1.69$, n.s., nor did the two variables interact, $F(1,38)=.48$, n.s..

Of greater interest was the relative difference between ratings provided for the male vs. female target obtained by subtracting the female-target from the male-target score. There was absolutely no difference between male and female participants but there was considerable inter-individual variance $(\mathrm{M}=.01, \mathrm{SD}=.72$ with a possible range -5 to +5$)$. . Thirty-eight percent of the sample considered males as more agentic than females (difference score $>.3$ ), $35 \%$ judged males and females as approximately equally agentic (difference score between .3 and +3 ), and $28 \%$ judged females as more agentic than males (difference score below -.3 ). These three subgroups represent three distinct types of participants associated with specific hypothesized positioning behaviours. Only those perceiving males as more agentic were expected to also position males to the left. We expected no bias for the participants who did not distinguish males and females in terms of agency, but a reversed bias for participants who endorsed a counterstereotypical representation of gender, with females being perceived as more agentic than males.

Left-positioning. We first tested whether respondents would show an overall tendency to position male targets to the left more often than would be expected by chance. Values ranged from 0 (all females but no males positioned to left) to 4 (all of the males and none of the females positioned to left), with 2 representing a chance distribution (two males and two females to left). On the average, participants positioned males to the left about half of the 
Groups in space

time $(M=2.23$ out of 4$)$, a value that did not differ reliably from what would be expected by chance, one-sample $t(39)=.94$, n.s.. Hence, there was no bias in the overall positioning of males or females to either the left or the right side of the scene, providing no support for a generalized positioning bias.

However, we had hypothesized that perceived agency would be predictive of left positioning, which was confirmed, $r(49)=.39, p<.05$, indicating that the more agency participants attributed to males (compared to females) the greater the tendency to position males (rather than females) to the left. This result is better understood by looking at the behavior of the three subgroups previously identified. A one-way ANOVA using the three sub groups as the independent and left positioning as the dependent variable, revealed a reliable effect, $F(2,37)=3.81, p<.05, \eta_{\mathrm{p}}{ }^{2}=.17$, which is represented in Figure 1 . Those that attributed greater agency to males tended to position males to the left more frequently than would be expected by chance $(M=2.80)$, one-sample $t(15)=2.35, p<.05$. The spatial positioning of those that attributed equal agency to males and females $(M=2.36)$ was no different from what would be expected by chance, one-sample $t(13)=.84$, n.s., whereas those who perceived females as more agentic tended to position males less frequently to the left than would be expected by chance $(M=1.27)$, although the difference from chance fell short of significance, one-sample $t(10)=-1.90, p<.09^{3}$.

\section{Discussion}

Paralleling the media analysis in Study 1B, the second study found the predicted link between the endorsement of traditional gender stereotypes that associate males with greater agency and the left positioning of males compared to females. Although many of our participants did not endorse the traditional gender stereotype, those that did tended to also place males to the left of females. Interestingly, participants who held counterstereotypic views showed the opposite tendency, placing women predominantly to the left. 
Groups in space

Considering that agency has generally been found to be an essential part of the male stereotype (Spence \& Helmreich, 1978; Spence, Helmreich, \& Stapp, 1974; Abele, 2003), it may surprise that our sample held such heterogeneous gender attitudes. One possible explanation is that the majority of our participants was young, had a relatively high educational level and was employed, thus representing a socioeconomic group in which traditional gender stereotypes may be less common than in the population at large.

\section{Study 3: Italian-Arabic comparison}

Our last study was conceptually and methodologically similar to Study 2, but we pursued two additional goals. First, we were interested to see whether the findings obtained for male and female targets would also generalize to other social groups that are stereotypically associated with different levels of agency. Thus, in addition to juxtaposing men and women, we also compared young adults and elderly people, assuming that younger adults would be associated with greater agency. Second, and theoretically more importantly, we intended to address the underlying reasons of the spatial agency bias. Specifically, we wanted to test whether the spatial positioning bias is a function of scanning habits that are linked to the way in which the dominant language is written in a given culture. We therefore compared students from Italy and from Arabic countries, since Italian is written from left to right, Arabic from right to left. If imaginary spatial trajectories are determined by scanning habits, then Italian speakers should position the more agentic group to the left, Arabic speakers to the right. Note that both Italian and Arabic are subject-verb-object (SVO) languages in which the sentence subject (in the majority of cases the Agent) precedes the sentence object (in the majority of cases the Patient).

\section{Method}

Participants. Sixty-one males participated in this research, including 30 Italian participants (mean age 24.40) and 31 participants who came from different Arabic countries (Lebanon, Morocco, and Syria, mean age 24.12), whose native language was Arabic and who 
Groups in space

were currently living in Northern Italy. The inclusion of only males was dictated by the fact that we were unable to find women in the region whose native language was Arabic. Since we intended to match the samples as closely as possible on socio-demographic variables, we decided to include only males in both cases. Approximately two thirds were students at the Universities of Padova and of Ferrara, the remaining third was employed in different professions All participants from Arabic countries were also fluent in Italian, but their native language was Arabic. The native language of all Italian participants was Italian and none of them had learned a RL language.

Procedure and Material. The study was introduced as concerning "how people form visual imagines of simple every day events". Participants were asked to complete an 8 page booklet, similar to the one described for Study 2. For each scene, participants received a simple drawing and were asked to add the two teams, indicating clearly where the two teams were positioned. On half of the vignettes, females and males were competing, on the other half young adults and elderly people. Example: "For the opening celebration of a new Youth Center, a chess tournament has been organized in which boys and girls compete. Please, indicate which side of the table the two teams are seated". Order of presentation of the scenes, order of presentation of gender- vs. age-based teams, and order of mentioning of the agentic (male, young) vs. non-agentic group(female teams competing egainst male teams vs. male teams competing against female teams) were fully counterbalanced across participants. This was essential since previous research (Chatterjee et al., 1999; Maass \& Russo, 2003; see also Study 2) indicated systematic order effects such that the first mentioned person is envisaged to the left. Subsequently, two scales were administered measuring how much agency participants attributed to males vs. females and to young versus old adults.

Importantly, the entire material (instructions, cartoons, agency scale, sociodemographic information) was presented in Italian or Arabic language, depending on the participant's native language. The translation from Italian to Arabic was done by a 
Groups in space

professional interpreter. After completion, participants were debriefed about the purpose of the experiment.

Spatial positioning. The most important measure was the spatial positioning of males vs. females and young vs. old adults in the cartoon scenes. For both social categories (males vs. females, young vs. old), values could range from 0 to 4 , with higher scores indicating that the more agentic group (men and young) was drawn to the left. The scale midpoint (2) corresponded to chance, meaning the complete absence of spatial bias.

Perceived Agency scale. Subsequently, the participants completed a modified, reduced, and translated version of Spence and Helmreich's (1978) Personal Attributes Questionnaire (of which only the masculine and the masculine-feminine items, constituting the agency subscale, were of interest) plus 3 items taken from the Dynamism subscale of Mulac's (1975, see also Mulac \& Lundell, 1986) Speech Dialect Attitudinal Scale. Based on a pretest on Italian- and Arabic-speakers, intended to assure equal meanings for each item for both languages, the 14 items that entered into the final agency scale were: independent, submissive ( $\mathrm{R}=$ reverse scoring), needful of the approval of others $(\mathrm{R})$, feelings easily hurt (R), can make decisions easily, gives up easily (R), self-confident, feels superior, strong need for security (R), stands up well under pressure, strong, active, fragile (R), remissive (R).

Participants completed the scale twice, one time rating men vs. women, the other rating young vs. old. The order of the scales was counterbalanced. For each item, participants were asked to indicate whether the characteristic was more typical of men or women (young or old) on a 6 point scale in which "men" and "women" (or, respectively "young" vs. "old") were the two endpoints. The internal consistency was .63 for the Men-Women and .55 for the Young-Old Agency scale (alpha for Italian: .73 and .47; for Arabic: .56 and .60). The reliability for the age category was relatively low, presumably because the scale was originally designed for evaluating gender, not age.

\section{Results}


Groups in space

Perceived agency. We had hypothesized that males would be perceived as more agentic than females and young people as more agentic than old people. In line with hypotheses, males were considered as more agentic than females (relative ratings $M=3.92$ ), compared to the neutral scale point of 3.5 , one-sample $t(60)=6.77, p<.001$, and young people as more agentic than old ones $(M=3.78)$, one-sample $t(60)=4.55, p<.001$. Importantly, males were perceived as relatively more agentic by both Italian- $(M=3.98), t$ $(29)=5.00, p<.001$, and Arabic-speaking participants $(M=3.87, t(30)=4.55, p<.001)$, and there was no difference in the stereotypic perception between the two groups, $t(59)=.88$, n.s.. Along the same line, young people were perceived as relatively more agentic by both Italian- $(M=3.72), t(29)=2.56, p<.05$, and Arabic-speaking participants $(M=3.84), t(30)$ $=3.85, p<.001$, with no reliable difference between groups, $t(59)=-.97$, n.s.. Together, these results suggest that stereotypic perceptions were indeed as expected, with males being perceived as more agentic than females and young people as more agentic than old people. Importantly, Arab and Italian participants showed highly similar stereotypic perceptions of these social categories.

Also, differently from Study 2, stereotypes were widely shared in our sample, with the large majority of participants endorsing traditional gender and age stereotypes. In fact $83 \%$ of the Italian-speakers and $84 \%$ of the Arabic-speakers considered males/young people as more agentic than females/old people.

Spatial positioning. We first ran a preliminary 2 (native language: Italian vs. Arabic) x 2 (category: male/female vs. young/old) x 2 (order of mentioning: agentic group first vs. second) ANOVA in which the last two factors were repeated measures, using the spatial positioning score as the dependent variable. This analysis showed that category had no effect either in itself or in interaction with the remaining variables (all F's $<1$ ), suggesting that findings for males vs. females did not differ from those for young vs. old people. We therefore collapsed the two target categories. 
Groups in space

A 2 (native language: Italian vs. Arabic) x 2 (order of mentioning: agentic group first vs. second) ANOVA with repeated measures on the second variable revealed two effects. First, a main effect for native language, $F(1,59)=5.22, p=.03, \eta_{\mathrm{p}}{ }^{2}=.08$, revealed a significant difference in the positioning between the two groups. Italian-speakers showed a tendency to draw the more agentic group (either males or young people) to the left $(M=$ 2.22), a value that differed from chance (2), one-sample t $(29)=1.61, p<.05$, one-tailed. In contrast, Arabic-speakers tended to draw the more agentic group to the right $(M=1.76)$, a value that differed from chance $(2)$, one-sample $\mathrm{t}(30)=1.63, p<.05$, one-tailed.

In addition, an interaction emerged between nationality and order of mentioning, $F$ (1, 59) $=11.96, p<.001$, partial $\eta_{\mathrm{p}}^{2}=.17$, that shows that the tendency of Italians to position the more agentic group to the left and the tendency of Arabs to position the same groups to the right was enhanced when the agentic group was mentioned first but disappeared when it was mentioned last. Indeed, one-sample t-tests (comparing the means with the neutral scale midpoint of 0 ) indicated that the left positioning bias of Italians differed reliably from zero when the agentic group was mentioned first $(M=.35)$, one-sample $t(29)=3.53, p<.001$. Conversely, the right-positioning bias in Arabs differed reliably from zero when the agentic group was mentioned first $(M=-.26)$, one-sample $t(30)=-2.28, p<.05$. When the less agentic group was mentioned first, means were no different from zero (Italians: $M=-.13$, Arabs: $M=.02$ ), suggesting the absence of a spatial positioning bias.

\section{Discussion}

The third study demonstrates a systematic spatial agency bias in both language groups, but in opposite directions. Italian-speakers tend to depict the more agentic group to the left, Arabic-speakers to the right. This result is nicely in line with the idea that spatial bias in the representation of groups is determined by scanning habits that pervade tasks that are unrelated to writing. 
Groups in space

This study also suggests that the spatial agency bias is not specific to males and females, but generalizes to other groups, such as young and old, that are perceived as differing in activity level. Finally, this last study also confirms the importance of word order in spatial representations. In line with previous work, there is an overwhelming tendency to imagine the target that was mentioned first, in the position in which writing starts (that is left for Italian-, and right for Arabic-speakers). Yet, this well-known order effect does not completely overrule the spatial agency bias, considering that when men or young people are mentioned first they are positioned to the left, but when women or old people are mentioned first they do not appear in the agentic left position any more frequently than would be expected by chance. Thus, the two phenomena (perceived agency and word order) seem to affect spatial positioning additively, both mirroring the culturally determined scanning habit.

\section{General Discussion}

In reference to Chatterjee (2002), we have proposed the existence of a SAB according to which, in LR writing cultures, action is perceived as evolving from LR and Agents being envisaged to the left of Patients. Since some social groups are perceived as more agentic than others, we hypothesized that our visual images of group interactions would reflect this bias, such that members of stereotypically more agentic groups would be envisaged to the left of those belonging to less agentic groups. In support of this idea, we found that Adam was generally portrayed to the left of Eve (Study 1a). We also found that those Italian speakers who did endorse traditional gender and age stereotypes with respect to agency positioned males to the left of females and young people to the left of old people (Study 3).

May this bias simply represent what we "see" in real life? For example, if mixed sex couples in Western cultures generally walk in a way that women are to the left of men (women's right shoulder being close to man's left shoulder), then the spatial agency bias could easily be explained as reflecting the actual spatial arrangement of men and women in daily life. Psychological research on spatial behaviors suggests otherwise. If anything there is 
Groups in space

a tendency for females to walk to the right of males. For example, Borden and Homleid (1978) observed that right-handed heterosexual couples arranged themselves so that significantly more females were on the males' dominant (right) side than would be expected by chance. Thus, it is highly unlikely that the SAB is in any way related to the spatial behavior of males and females, as we observe it in everyday situations.

We had also advanced a second prediction, namely that the SAB would emerge only when targets were indeed associated with differential degrees of agency. In Study 1b we compared the spatial positioning of male-female pairs of three TV series (the Simpsons, the Flintstones, the Addams) and found evidence for the SAB only for the couple (the Addams) for which independent raters - familiar with the three series - had indeed indicated that the male was more agentic. Conceptually similar are the results of Study 2 where we found evidence for the SAB only for those participants who believed that males, as a group, are more agentic than females, but a (non-significant) reversal for those who considered females to be the more agentic group. A complete absence of spatial bias was observed for those that denied gender differences in agency. Together, these two studies (Study 1b and Study 2) suggest that the SAB is indeed linked to stereotypic expectancies. Atypical members of agentic groups, such as Homer Simpson, are unlikely to be positioned to the left and, by the same logic, observers who do not endorse the stereotype fail to show the SAB. Unfortunately, we were unable to test this same hypothesis is Study 3 because the vast majority of our allmale sample endorsed traditional stereotypes.

The third important finding of our set of studies regards the underlying reasons of the SAB. Comparing Italian and Arabic speaking participants, we found that both groups show systematic spatial biases, but in opposite directions. This is exactly what one would expect if one gives credence to the cultural hypothesis according to which scanning habits, possibly combined with standard ordering of subject (Agent) and object (Patient), lead to a general LR or RL scheme of action. 
Groups in space

Together, the results are consistent with hypotheses, yet they are of small magnitude. This is in line with other findings in this research area. For instance, gender biases in portrait orientation generally become visible only when analyzing very large samples of art work. Similarly, in our own research project we have found spatial biases in adult populations in different areas (advertising, political campaigns), but generally these biases are small and malleable (although we generally do not find any reliable reversals). This lack of strength is annoying from a researcher's point of view, but reassuring from an applied perspective. As argued by Anjan Chatterjee (personal communication) it would be dysfunctional for survival if the human mind was rigidly programmed to perceive actions with a specific trajectory or to systematically associate certain social groups with specific spatial positions. Given that our experience is constantly embedded in space, a stable and pervasive SAB would not allow us to interact flexibly with our physical and social environment. As a consequence, the subtlety of horizontal spatial bias is not only unsurprising, but also desirable.

Open questions

Not surprisingly, many questions remain open at this point. First of all, it remains to be seen whether motor and visual habits related to writing and reading are sufficient to explain the obtained results, or whether the standard ordering of sentence subject and object are critical in the development of a spatial schema of action.

If linguistic factors play a role, as we suspect, then the SAB should only occur in languages in which the Agent precedes the Patient in standard active phrases (e.g. Fabio feeds the dog). In languages like English, Italian, French, German, and many others, the Agent (Fabio in our example) typically occurs in the subject role, unless the verb appears in the passive tense (The dog was fed by Fabio). Indeed, in many languages (such as English) the relative ordering determines Agent vs. Patient assignment (Fabio feeds the dog vs. The dog feeds Fabio). These languages, also referred to as nominative-accusative languages (Duranti, 
Groups in space

2004), should show a systematic bias in envisaging the agent either to the left or to the right, depending on writing direction.

However, there are languages in which the object tends to precede the subject. Also, some languages use other ways to encode Agency. There are languages such as Samoan, referred to as ergative-absolutive languages, in which the Agent is marked by a preposition (ergative marker), rather than being distinguished by position. In another group of languages, identified by Duranti (2004) as stative-active languages, roles are defined by verbs that either mark their subject as Agent or as Patient. Considering these variations across languages, it would be interesting to investigate whether $\mathrm{SAB}$ is limited to those languages in which Agents generally precede Patients in active sentences (although this word order may not necessarily be mandatory). It is possible that the findings reported here only hold for languages in which Agency is systematically associated with position (nominative-accusative languages), but not for those in which Agent and Patient are marked in different ways. Although we have currently no direct proof of this hypothesis, the question could be investigated by extending our paradigm to ergative-absolutive or to stative-active languages. Implications

In our opinion, the SAB may have a number of interesting implications. First of all, although the bias is of small magnitude, it may play a subtle role in different areas involving images, such as films or news reports. Although we have no data to support this idea, it is possible that film or theater directors inadvertently construct scenes so that the more active or stereotypically more agentic character is placed to the left of the receiving end of the action. Also, news reports may be biased so as to favor one of two opponents (for example in malefemale interactions) in terms of implicit agency. As a case in point, there are historical documents that testify to the fact that German military reporters showed German soldiers almost exclusively with a LR trajectory, as ordered by the Ministry of Propaganda in Nazi Germany (reported in Reitz' famous historical TV serial “Heimat”; see Buchmann, 2006). 
Groups in space

Thus, it remains to be seen whether spatial arrangements in films, news reports etc. are systematically related to stereotypic beliefs or, possibly, to the intention to make one party or the other appear more active or more influential.

Second, artistic production may well reflect socially shared stereotypes with painters and photographers placing the more agentic person to the left. The idea that artwork reflects social beliefs is by no means new. Just like social psychologists, art historians often interpret religious iconography as reflecting longstanding stereotypes. Interestingly, art historians often acknowledge the fact that differences between social categories may be exaggerated for the sake of the composition. As a case in point, Clifton (1999) who has analyzed Masaccio’s Expulsion from the Garden of Eden underlines the artists' use of the principle of contrapposto both in gesture and anatomy of the subjects that are part of the pictorial composition. This principle of contrapposto as evident in pictorial representations of Adam and Eve appears quite similar to the psychological opposition of agentic-instrumental vs. communalexpressive characteristics associated with males and females as originally proposed by Bakan (1966). In other words, pictorial representations such as Masaccio's and psychological theorizing such as Bakan's agree in assigning women characteristics such as passivity, modesty, and shame that are placed in opposition to the active and secure behaviors of males. Our research suggests that one subtle way to communicate differential agency in artwork lies in the spatial arrangement.

Third, one may hypothesize that self-presentations are not immune to the SAB. For example, it is conceivable that individuals with highly agentic self-concepts choose to present themselves to the left, facing right. First evidence comes from a study by Nicholls, Clode, Wood and Wood (1999) in which participants, regardless of gender, presented their left cheek when asked to pose for a family portrait and their right cheek when posing as scientists. It is therefore plausible that actors engage in particular spatial positioning in line with their selfstereotyping. Although we are not aware of any study investigating spatial arrangements of 
Groups in space

more than one person, it is conceivable that similar left-right symmetries may occur, with people preferring to be presented to the left of others if they want to display a high degree of agency.

At this point, one may wonder whether spatial arrangements such as those reported here really matter. Are observers sensitive to variations in R vs. L positioning? As far as portrait direction is concerned (see Nicholls et al., 1999) there is tentative evidence that observers form impressions that are in line with the (presumed) sitters' intention, considering that right-oriented portraits are rated as more "scientific" (and presumably less emotional) than left-oriented ones (ten Cate, 2002, Study 2). Another research, that speaks more directly to the implicit agency of L vs. R-oriented action, has recently been conducted by Maass, Pagani, and Berto (2007). These authors found that Italian speakers perceived the same athletic performance (a soccer goal) as stronger, faster, and more beautiful if presented with a LR rather than RL trajectory. Participants also interpreted aggressive film scenes as more violent and more harmful to the victim when shown with a left-to-right trajectory. Thus, there is first evidence that observers may indeed be sensitive to the implicit meaning of different spatial arrangements.

\section{Conclusion}

Although many questions remain necessarily open at this point, this set of studies suggests that there is a subtle spatial bias that, to our knowledge, has not been identified by previous research on intergroup relations. Though of small magnitude, this bias seems to pervade spatial imaging as well pictorial representations, art work etc. in a systematic way. Although people may not be aware of this bias, and, indeed, may not even rationally reflect on their spatial decisions, they seem to apply a spatial schema of action that is consistent with culturally determined scanning habits. 
Groups in space

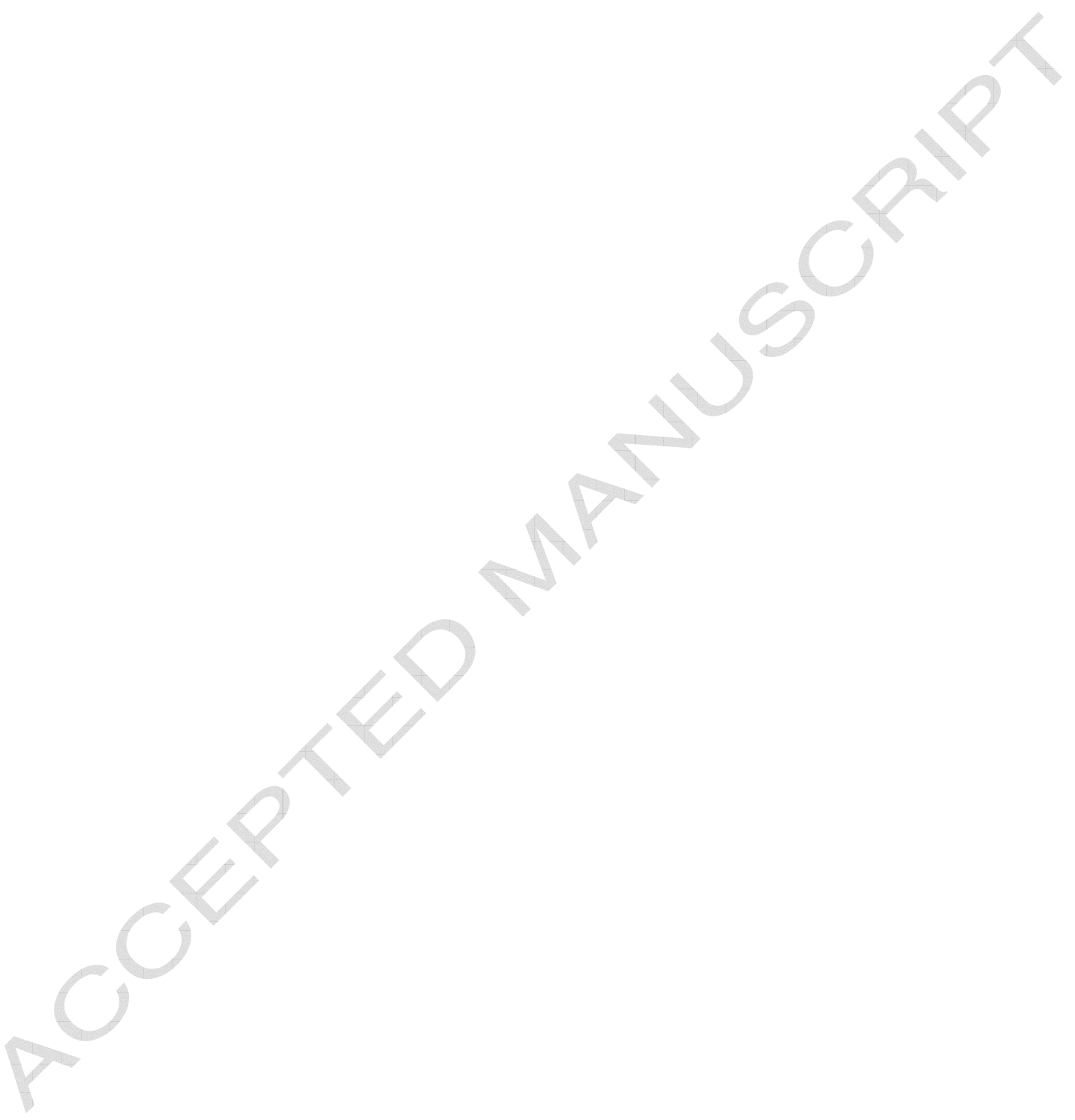




\section{References}

Archer, D., Iritani, B., Kimes, D. D., \& Barrios, M. (1983). Face-ism: Five studies of sex difference in facial prominence. Journal of Personality and Social Psychology, 45(5), 725-735.

Abele, A.E. (2003). The Dynamics of Masculine-Agentic and Feminine-Communal Traits: Findings From a Prospective Study. Journal of Personality and Social Psychology, 85 (4), 768-776.

Bakan, D. (1966). The duality of human existence. Chicago: Rand Mc Nally.

Barsalou, L. W. (1999). Perceptual symbol systems. Behavioral and Brain Sciences, 22(4), $577-660$.

Borden, R. J. \& Homleid, G. M. (1978). Handedness and lateral positioning in heterosexual couples: Are men still strong-arming women? Sex-Roles, 4, 67-73.

Boroditsky, L. (2001). Does language shape thought?: Mandarin and English time: speakers' conceptions of time. Cognitive Psychology, 43, 1-22

Buchmann, W. (2006). Bilder in Archiven [Pictures in archives] http://www.bundesarchiv.de/imperia/md/content/abteilungen/abtb/bildarchiv2/1.pdf

Chatterjee, A. (2001). Language and space: some interactions. Trends in Cognitive Science, 5, $55-61$.

Chatterjee, A. (2002). Portrait profiles and the notion of agency. Empirical Studies of the Arts, 20, 33-41.

Chatterjee, A., Southwood, M. H., \& Basilico, D. (1999). Verbs, events and spatial representations. Neuropsychologia, 395-402.

Chokron, S., \& De Agostini, M. (2000). Reading habits influence aesthetic preference. Cognitive Brain Research, 10, 45-49. 
Groups in space

Clifton, J. (1999). Gender and Shame in Masaccio's Expulsion from the Garden of Eden. Art History, 22, 637-55.

Conway, M., Pizzamiglio, M.T., \& Mount, L. (1996). Status, Communality, and Agency: Implications for Stereotypes of Gender and Other Groups. Journal of Personality and social Psychology, 71-1, 25-38.

Dehaene, S., Bossini, S. \& Giraux, P. (1993). The mental representation of parity and number magnitude. Journal of Experimental Psychology: General, 122 (3), 371-396.

Duranti, A. (2004). A Companion to Linguistic Anthropology. Blackwell Publishing Ltd, Oxford.

Elkind, D. \& Weiss, J. (1967). Studies in perceptual development, III: Perceptual exploration. Child Development, 38, 553-561.

Else-Quest, N. M., Hyde, J. S., Goldsmith, H. H., \& Van Hulle, C. (2006). Gender differences in temperament: A meta-analysis. Psychological Bulletin, 132 (1), 33-72.

Fagard, J. \& Dahmen, R. (2003). The effects of reading-writing direction on the asymmetry of space perception and directional tendencies: A comparison between French and Tunisian children. Laterality, 8, 39-53.

Fiske, S. T., Cuddy, A. J., Click, P., \& Xu, J. (2002). A model of (often mixed) stereotype content: Competence and warmth respectively follow from perceived status and competition. Journal of Personality and Social Psychology, 82, 878-902.

Fiske, S. T., Xu, J., Cuddy, A. J. C., \& Click, P. (1999). (Dis)respecting versus (dis)liking: Status and interdependence predict ambivalent stereotypes of competence and warmth. Journal of Social Issues, 55, 473-491.

Freyd, J. J., \& Finke, R. A. (1984). Representational momentum. Journal of Experimental Psychology: Learning, Memory, \& Cognition, 10, 126-132.

Gevers, W. Reynvoet, B., \& Fias, W. (2004). The mental representation of ordinal sequences is spatially organized: Evidence from days of the week. Cortex, 40, 171-172. 
Groups in space

Gordon, I. (1974). Left and right in Goya’s portraits. Nature, 294, 197-198.

Grüsser, O. J., Selke, T., \& Zynca, B. (1988). Cerebral lateralization and some implications for art, aesthetic perception and artistic creativity. In I. Rentschler, B. Herzberg, \& D. Epstein (Eds.), Beauty and the brain: Biological aspects of aesthetics (pp. 257-293). Boston: Birkhauser.

Halpern, A. R., \& Kelly, M. H. (1993). Memory biases in left versus right implied motion. Journal of Experimental Psychology: Learning, Memory, \& Cognition, 19, 471-484.

Heron, W. (1957). Perception as a function of retinal locus and attention. American Journal of Psychology, 70, 38-48.

Hubbart, T. L. (2005). Representational momentum and related displacement in spatial memory: A review of the findings. Psychonomic Bullettin and Review, 12 (5), 822-851.

Humphrey, N. K. , McManus, I. C. (1973). Status and the left cheek. New Scientist, 59, 437439.

Kolbe, R., \& Albanese, P. (1996). Man to man: A content analysis of sole-male images in male-audience magazines. Journal of Advertising, 35(4), 1-20.

Jackendoff, R. (1996). The architecture of the linguistic-spatial interface. In Bloom et al. (Eds.) Language and Space, Cambridge, MA: The MIT Press (pp. 365-384).

Judd, C. M., James-Hawkins, Yzerbyt, V., \& Kashima, Y. (2005). Fundamental dimensions of social judgment: Understanding the relations between judgments of competence and warmth. Journal of Personality and Social Psychology, 89, 899-913.

Maass, A., \& Russo, A. (2003). Directional bias in the mental representation of spatial events: Nature or Culture? Psychological Science, 14 (4), 296-300.

Maass, A., Pagani, D., \& Berta, E. (2007). How beautiful is the goal and how violent is the fistfight? Spatial bias in the interpretation of human behavior. Social Cognition, 25(6), 833-852. 
Groups in space

McGuire, W. J. \& McGuire, C. V. (1992). Psychological significance of seemingly arbitrary word-order regularities: The case of kin pairs. In Semin, Gün R.; Fiedler, Klaus (1992). Language, interaction and social cognition. (pp. 214-236). Thousand Oaks, CA, US: Sage Publications.

McManus, I. C. (2005). Symmetry and asymmetry in aesthetics and the arts. European Review, 13 (2), 157-180.

McBeath, M. K., Morikawa, K., \& Kaiser, M. K. (1992). Perceptual bias for forwardfacing motion. Psychological Science, 3, 362-367.

Mulac, A. (1975). Evaluation of the speech dialect attitudinal scale. Speech Monograph, 42, 182-89.

Mulac, A., \& Lundell, T. L. (1986). Linguistic contributors to the gender-linked language effect. Journal of Language and Social Psychology, 5, 81-101

Nachshon, I. (1985). Directional preferences in perception of visual stimuli. International Journal of Neuroscience, 25, 161-174.

Nachshon, I., Argaman, E., \& Luria, A. (1999). Effects of directional habits and handedness on aesthetic preference for left and right profiles. Journal of Cross-Cultural Psychology, 30, 106-114.

Nicholls, M. E. R., Clode, D., Wood, S. J., \& Wood, A. G. (1999). Laterality of expression in portraiture: Putting your best cheek forward. Proceedings of the Royal Society of London. Series B, 266, 1517-1522.

Richardson, D.C., Spivey, M.J., Barsalou, L.W., McRae, K. (2003). Spatial representations activated during real time comprehension of verbs. Cognitive Science, 27, 767-780.

Santiago, J., Pérez, E., Lupiáñez, J., \& Funes, M. J. (2007). Time (also) flies from left to right. Psychonomic Bulletin \& Review, 14, 512-516

Schwarz, N., \& Kurz, E. (1989). What's in a picture? The impact of face-ism on trait attribution. European Journal of Social Psychology, 19(4), 311-316. 
Groups in space

Smith, L.B. (2005). Cognition as a dynamic system: Principles from embodiment. Developmental Review, 25, 278-298.

Spalek, T. M. \& Hammad, S. (2004). Supporting the attentional momentum view of IOR: Is attention biased to go right? Perception \& Psychophysics, 66 (2), 219-233.

Spence, J.T., Helmreich, R. (1978). Masculinity and femininity: Their psichological dimensions, correlates and antecedents. Austin, University Press.

Spence, J.T., Helmreich, R., \& Stapp, J. (1974). The Personal Attributes Questionnaire: A measure of sex role stereotypes and masculinity-femininity. JSAS Catalog of Selected Document in Psychology, 4, 43-44.

Suitner, C \& Maass, A. (2007). Positioning Bias in Portraits and Self-Portraits: Do Female Artists make different choices? Journal of Empirical Studies of the Arts, 25 (1), 71-95. ten Cate,C. (2002). Posing as a professor: Laterality in posing orientation for portraits of scientists. Journal of Nonverbal Behavior, 26 (3), 175-192.

Tversky, B., Kugelmass, S. \& Winter, A. (1991). Cross-cultural and developmental trends in graphic productions. Cognitive Psychology, 23, 515-557. 
Groups in space

\section{Author Note}

Correspondence regarding this article should be addressed to Anne Maass,

Dipartimento di Psicologia dello Sviluppo e della Socializzazione, Università di

Padova, Via Venezia 8, 35139 Padova

Italy, e-mail: anne.maass@ unipd.it. 


\section{Footnotes}

1. For instance, focussing exclusively on books that are only a small portion of printed material, British citizens spend approximately 5.5 hours per week reading, with women (6,7 hours) reading more than men (4,2 hours). Reading statistics for European countries can be found in the following website:

http://www.readingeurope.org/observatory.nsf/

2. An additional gender identification scale produced no effect and will therefore not be discussed further.

3. There also was an effect for order of presentation with the first mentioned group being placed to the left more often than would be expected by chance.

However, since this effect did not interact with any other variable, this finding is of little theoretical relevance in this context.

4. Results were practically identical when splitting the sample only in two groups (above vs. below scale midpoint). In this case, those who perceive males as more agentic also position males predominantly to the left $(M=2.79)$, which differed significantly from chance, one-sample $t(23)=2.87, p=.009$, whereas those perceiving females as more agentic, showed a tendency to position females to the left $(\mathrm{M}=1.38)$, one-sample $t(15)=-1.84, p=.086$. 
Groups in space

\author{
Figure Caption
}

Figure. Spatial positioning of males as a function of agency-related stereotypic beliefs (Exp. 2). 


\section{Groups in space}

$\underline{\text { Figure }}$

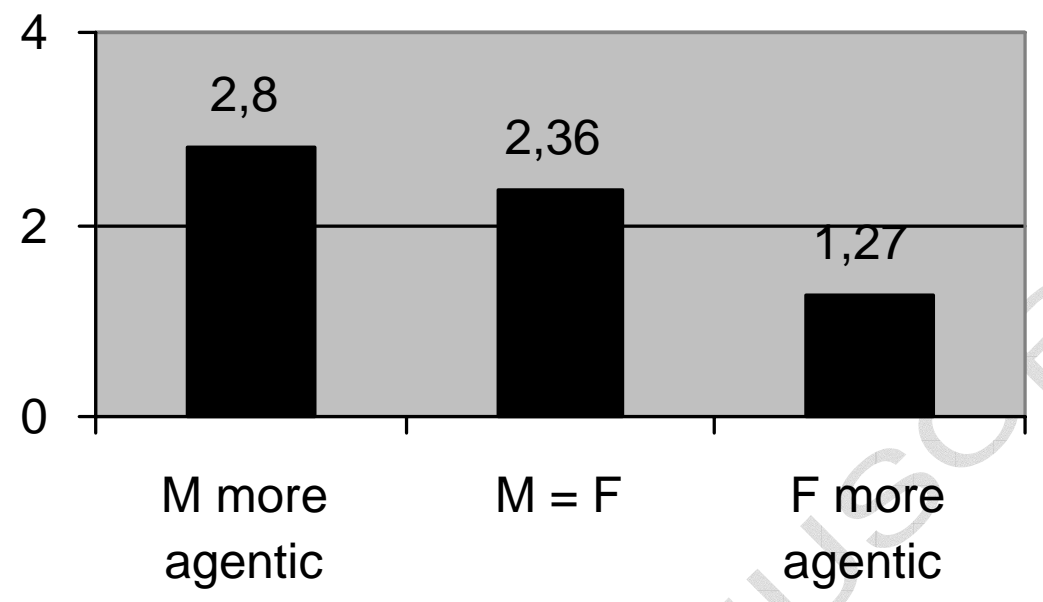

Agency-related stereotypic beliefs 